\title{
DUST CONDENSATION IN STELLAR OUTFLOWS
}

\author{
E. SEDLMAYR \\ Institut für Astronomie und Astrophysik, Technische Universität Berlin
}

\begin{abstract}
The basic concepts of astrophysical dust formation are surveyed. The potentials, the limitations and the shortcomings of the various approaches are discussed.
\end{abstract}

\section{INTRODUCTION}

If a multicomponent system consisting of atoms, ions and molecules becomes cool enough particular molecules begin to cluster and finally macroscopic solid particles are formed. This process of nucleation and grain growth is well known to occur in the stellar outflows of cool red giants and supergiants, and also in novae and supernovae ejecta, manifesting itself in a significant continuum IR excess and in a variety of spectral features which are assumed to be characteristic for the absorption or emission of photons by macroscopic solid particles. But since the analysis of such observations yield only information about the interaction of photons with special functional groups of the grains (cf. Gail and Sedlmayr, 1987a) definitive conclusions concerning the true chemical and crystalline structure of the grains are not directly possible. Only for carbon grains, however, the observed $\lambda^{-1}$-dependence of continuous extinction (e.g. Jura, 1983; Rowan-Robinson and Harris, 1983; Tanabe, 1983; Rengarajan, 1985) suggests particles with "amorphous" lattice structure distinct from pure graphite, which would show a $\lambda^{-2}$ behaviour (Koike et al., 1980; Wirsich, 1987). Moreover, for Mie-particles with a size smaller than the wavelengths of the interacting photons the extinction is given by the total amount of condensed material, hence no information about the definite size of the grains is provided by IR observations in this case.

For these reasons a detailed physical and chemical investigation of the basic mechanisms of grain formation and growth is necessary to arrive at a consistent description of a dust forming astrophysical system. With regard to such an $a b$ initio study stellar outflows represent very interesting candidates for the following reasons:

- there exists a large amount of observational material covering the relevant spectral regimes,

- a sufficient number of model calculations, including radiative transfer and hydrodynamics are available, and

- number densities of relevant molecules can be calculated, at least under the assumption of chemical equilibrium. 
A very important point in this context is also that the dust observed in stellar outflows (which is considered to represent a major source of seed particles for the various dust components of the interstellar medium) cannot be primordial. It has to originate from the gas phase somewhere in the flow where the conditions are favourable, thus providing well defined initial conditions deep inside the shell where the temperature is too high to allow solids to exist.

It is the aim of this contribution:

- to present some basic descriptions of astrophysical grain formation and growth,

- to discuss their relevance for concrete situations, in particular for the shells of cool giants and supergiants, and

- to point out the potentials, the limitations, and the shortcomings of these methods.

\section{THEORETICAL DESCRIPTION OF GRAIN FORMATION AND GROWTH}

Solid particles in a gaseous medium can, in principle, be considered as the products of a large number of "constructive" chemical reactions resulting finally in macroscopic specimens. Such a fundamental microscopic description of grain formation, however, is currently far from tractable, not only due to the huge reaction network required, but also because of the lack of most relevant input parameters (see for instance the set of kinetic equations in Donn et al., 1981). Fortunately, because of the existence of a well defined condensation barrier, expressed by a corresponding critical cluster size, the formation of solid particles in a gaseous medium, can be roughly considered as a two-step process:

1) the formation of critical clusters, and

2) the growth of these clusters to macroscopic dust grains.

The latter process, in principle, can be treated thermodynamically by means of a master equation, which yields the entire time-dependent local size distribution function. For this reason the growth of the particles can easily be calculated if the interactions of the grains with the colliding molecules (i.e., cross sections, sticking coefficients, reaction probabilities etc.) are known (cf. Sect. 2.2). Hence the most severe problem of the gas-solid transition is the nucleation problem, i.e., to determine the critical clusters and to provide a reliable quantitative description of their formation.

\subsection{THE NUCLEATION OF CRITICAL CLUSTERS}

In astrophysical problems the critical clusters for the condensates expected are very small, with $N$ the number of constituent monomers on the order of 10 . Hence only a few chemical reactions are required for a cluster to surmount the condensation barrier. For the conditions prevailing in stellar outflows these chemical reaction timescales usually are very small compared to the characteristic timescales governing the hydrodynamic and thermodynamic evolution of the medium. Thus the formation of critical clusters in stellar outflows can be considered as a stationary process (Gail and Sedlmayr, 1988; Rossi and Benevides-Soares, 1988), with the exception of shock fronts, which are not considered in the context of this paper. 
Besides the work of Yamamoto and Nishida, 1977, who treat the nucleation phase by solution of a master equation (a method which due to its formidable numerical effort, is practically restricted to few special applications), until now, only two limiting approaches are available for describing the formation of critical clusters: 1 . classical nucleation theory, and 2. the construction of individual chemical pathways from the molecules to macroscopic specimens.

\subsubsection{Classical nucleation theory}

Classical nucleation theory describes the formation of critical clusters in a supersaturated vapour by means of thermodynamic quantities considering the clusters in the nucleation regime as solid particles. The properties of the particles are described either by extrapolation of the corresponding bulk phase quantities, even into the domain of very small clusters, or by interpolation between the bulk phase and the corresponding molecular values (cf. Salpeter, 1977; Draine and Salpeter, 1977; Draine, 1979).

As these procedures do not take into account the individual properties of the considered clusters (e.g. shape, internal structure,...) the thermodynamic properties derived in this way might be severely in error or even ill defined, e.g. the attribution of a surface tension to a cluster consisting of only few monomers. Therefore, it remains a formidable, and, in many cases, an unsolved problem, to determine realistic thermodynamic functions (entropy, enthalpy) for the clusters in the nucleation regime. On the other hand, these methods yield analytical expressions both for the thermodynamic quantities and the rate coefficients as function of the cluster size $N$, and thus allow a straightforward calculation of the stationary nucleation rate (see for instance the review of Feder et al., 1966).

In classical nucleation theory three different situations have to be distinguished:

\section{i) Homomolecular homogeneous nucleation}

This mechanism describes the formation of homogeneous clusters by direct condensation of molecules of one kind (monomers). In this case, and for thermal equilibrium, the stationary nucleation rate is, according to Becker and Döring (1935), immediately given by (cf. Gail and Sedlmayr, 1988)

$$
J_{*}=\left[\sum_{i=1}^{\hat{N}} \frac{\tau_{g r, i}}{\circ}\right]^{-1}
$$

with $\stackrel{\circ}{f}(i)$ being the local equilibrium number density of clusters of size $i$ (i. e., consisting of $i$ monomers) and $\tau_{g r, i}$ the corresponding growth timescale for the addition of monomers

$$
\tau_{g r, i}=\frac{1}{A_{i} \beta_{i}}
$$

where $\beta_{i}$ is the constructive monomer flux impinging onto an $i$-cluster and $A_{i}$ its surface area. $\hat{N}$ is an arbitrarily chosen cluster size well beyond the nucleation barrier defined by the maximum term $i=N_{*}$ in the sum of eqn. (1). As this 
term just describes the transition of the clusters through the "bottle neck", and therefore is decisive for the entire nucleation problem, the nucleation rate usually is calculated by considering these critical clusters only

$$
J_{*}=\frac{\stackrel{\circ}{f}\left(N_{*}\right)}{\tau_{g r, N_{*}}} \cdot \frac{1}{Z}
$$

where the Zeldovich factor $Z$ accounts for the fact that not only the critical clusters but also clusters in the vicinity of $N_{*}$ can grow or evaporate. For a general description of the problem including non-LTE effects see Gail and Sedlmayr, 1988. For an application of this method to study the primary condensates $(\mathrm{MgS}-, \mathrm{Fe}-$, and $\mathrm{SiO}$-clusters) in the outflow of M-Giants see Gail and Sedlmayr, 1986, 1987a.

\section{ii) Heteromolecular homogeneous nucleation}

This mechanism describes the formation of homogeneous clusters by reactions with various kinds of molecules including chemical sputtering. For example the formation of a pure carbon cluster by reactions with $C_{1}, C_{2}, \ldots, C_{2} H, C_{2} H_{2}$, etc.. Also in this case the nucleation rate can be calculated in a straight-forward manner by the Becker-Döring method.

Despite the widespread use of classical homogeneous nucleation theory in addressing astrophysical dust formation problems, the validity of using this approach is questionable, essentially for the following reasons (e.g. Donn et al., 1981; Donn and Nuth, 1985):

- the clusters are considered as macroscopic systems, the thermodynamic functions of which are calculated from bulk phase properties, even in the case of particles consisting of only few monomers,

- the surface tension concept becomes ill defined for the small critical clusters expected under astrophysical conditions,

- the geometrical shape is restricted to spherical particles, an assumption which does not account for the individual structure of clusters and large molecules, and,

- grain formation is a phase transition, which requires conditions far from thermal equilibrium to proceed in a finite time.

These shortcomings might be the reason for the rather high theoretical condensation temperature anticipated for carbon grains in C-star shells. A result which does not agree with the observations (cf. Gail and Sedlmayr, 1985).

\section{iii) Heterogeneous nucleation}

This mechanism describes the formation of clusters by chemical reactions with already existing kernals, resulting in particles of a new chemical composition. This process might be important for several astrophysical problems. Some examples include the formation of silicate grains in M-type stars (e.g. Gail and Sedlmayr, 1986; and the condensation experiments by the NASA group (see Nuth and Donn, 1987, 
and references therein)) or dust formation in novae and supernovae ejecta; and also in environments contaminated by UV-radiation where ions and charged clusters might play a major role in the nucleation process. Furthermore, heteromolecular accretion of molecules onto dust nuclei is supposed to be an important mechanism for dust processing in the interstellar medium (see for instance the review of Tielens and Allamandola, 1987 and references therein).

However, since heterogeneous nucleation, hitherto has not been studied in detail for astrophysical applications, we refrain from a further discussion of this mechanism (cf. Castleman, 1979; Draine, 1979).

\subsubsection{Chemical description of the molecule-solid-transition}

The aim of this method is to provide a chemical pathway from the molecules to the macroscopic particles by construction of a realistic reaction chain. This approach is purely microscopic and requires the detailed discussion of every reaction step, where the thermodynamic and kinetic properties of the molecules involved have to be known. Since this is a very elaborate task, such a procedure is limited to specific problems, for example soot formation in carbon rich environments (Keller, 1987; Gail and Sedlmayr, 1987a). In this problem one is guided by laboratory flame chemistry and the wealth of data provided by pyrolysis experiments (e.g. Frenklach et al., 1984).

In this way carbon nucleation in C-star shells is described by a chemical pathway starting at acetylene (which is the most abundant condensable species) and leading via a series of polycyclic aromatic hydrocarbons (PAHs) of increasing complexity to planar macroscopic structures (Keller, 1987; Gail and Sedlmayr, 1987a), which exhibit the typical emission features attributed to carbonaceous grains (Léger and Puget, 1984; Allamandola et al., 1987). An extensive review covering the various aspects of astrophysical carbon dust formation is provided by Tielens, 1988.

\subsection{THE GROWTH REGIME}

The growth of particles larger than the critical cluster size can be described by the time evolution of the local size distribution function $f(t, N)$ according to the corresponding master equation. Because the relevant timescales, at least for sufficiently large particles, usually are comparable or larger than the hydrodynamical timescales governing the evolution of the systems, the growth phase has to be treated as a time dependent problem. However, in most astrophysical situations it is not necessary to have the full information provided by the distribution function, but it suffices to use appropriate moments (cf. Gail and Sedlmayr, 1988):

$$
K_{i}=\sum_{N_{l}}^{N_{\star}} f(t, N) N^{i / \gamma}
$$

which directly enter into the model calculations. $N_{l}$ and $N_{u}$ are suitably chosen lower and upper limits of summation. $\gamma$ is the dimension of the considered cluster; $\gamma=2$ for planar and $\gamma=3$ for three-dimensional particles, respectively. 
By means of these moments the time evolution of the dust component is approximately given by the following coupled system of equations:

$$
\begin{aligned}
\frac{\partial K_{o}}{\partial t}+\operatorname{div}\left(\mathrm{v} K_{o}\right) & =J_{*} \\
\frac{\partial K_{i}}{\partial t}+\operatorname{div}\left(\mathrm{v} K_{i}\right) & =\frac{i}{\gamma \tau_{g r}} K_{i-1} \quad i=1,2, \ldots, i_{\text {max }}
\end{aligned}
$$

with $\tau_{g r}$ being the growth timescale, i.e., the timescale of constructive collisions of a dust particle with the condensing molecules, and $\mathbf{v}$ the hydrodynamic velocity of the flowing medium.

Eqn. (4) describes the evolution of the number density of grains due to the local production rate of critical clusters $J_{*}$ and eqns. (5) accounts for the growth of these clusters.

The truncation of the system (5) depends on the problem considered. For example in an astrophysical system where only Mie-absorption of the grains is important the use of $i_{\max }=3\left(i_{\max }=2\right)$ in the case of three-dimensional (two-dimensional) particles is reliable (e.g. Gail et al., 1984).

\subsection{THE GRAIN SIZE SPECTRUM}

On the basis of a given model of a dust-forming astrophysical system (calculated by the methods outlined above) it is straightforward to derive the grain size distribution for the case that evaporation can be neglected.

Assuming $f(t, N)$ to be a smooth function, the sum in eqn. (3) can be expressed by the integral

$$
K_{o}(t)=\int_{N_{l}}^{N_{u}} f(t, N) d N .
$$

Then from (4), for an element of matter moving along a streamline, it immediately follows that:

$$
d K_{o}(t)=d \int_{N_{l}}^{N_{u}} f(t, N) d N=J_{*} d t
$$

from which by functional integration and some simple manipulations the size distribution function

$$
f(t, N) d N=\int_{t(N)}^{t(N+d N)} J_{*} d t^{\prime}
$$

is obtained, where $t-t(N)$ is the time required for a critical cluster to grow up to size $N$. For a more detailed description of this procedure see Dominik et al., 1988.

In both the momentum method (Sect. 2.2) and the calculation of the size spectrum, coagulation of dust grains has been neglected. However, due to the lack of pertinent investigations, until now, it is not clear whether this process can play a significant role in stellar outflows. 


\subsection{MONOCRYSTALLINE, POLYCRYSTALLINE OR AMORPHOUS GRAINS}

In order to decide whether monocrystalline, polycrystalline or amorphous grains are produced by condensation in an astrophysical system, four different timescales have to be compared: a) the mean capture time of a cluster for a condensing molecule $t_{c}$, i. e., the creation time for admolecules adsorbed at the grain's surface, b) the hopping time of an admolecule $t_{h}, \mathrm{c}$ ) the time for an admolecule to find a suitable lattice site for being built in $t_{n}$, and d) the evaporation time $t_{e v}$ (see Gail and Sedlmayr, 1984). According to the relations between these timescales different crystalline structures of the grains have to be expected:

i) $t_{c}<t_{e v}:$ Grain growth takes place. This inequality always has to hold for macroscopic particles to be formed.

ii) $t_{n}=n^{2} t_{h}<t_{c}$ : The admolecule, after a random walk with $n^{2}$ steps, always arrives at the energetically most favourable lattice site to be built in. In this case monocrystalline grains are expected to form.

iii) $t_{n} \gtrsim t_{c}$ and $t_{h}<t_{c}$ : The grain cannot grow via a sequence of equilibriun configurations. Nevertheless, there exists still a short range order resulting in an irregular polycrystalline structure.

iv) $t_{h}>t_{c}$ : In this case amorphous grains are expected to form.

Following a typical wind trajectory of a cool giant or supergiant beyond the condensation barrier $\left(t_{c} \simeq t_{e v}\right)$ we therefore expect a monotonic transition via the situations ii) $\rightarrow$ iii) $\rightarrow$ iv), finally resulting in grains, consisting of a monocrystalline core, a polycrystalline shell and eventually an amorphous mantle (cf. Gail and Sedlmayr, 1984).

Such a scenario might explain the often observed $\lambda^{-1}$-behaviour of the circumstellar extinction in carbon stars attributed to a photon interaction with amorphous carbon and also the occurrence of "amorphous" silicates in M-type shells (cf. Dorschner et al., 1986, and references therein).

\section{CIRCUMSTELLAR CHEMISTRY}

In view of the preceding sections, dust formation is a genuine chemical problem which can be modelled only on the basis of a detailed knowledge of the molecular abundances and the kinetic behaviour of the system. This concerns not only the atoms and molecules directly involved in the condensation process (e. g. SiO, $\mathrm{MgS}$, $\left.\mathrm{MgO}, \mathrm{Fe}, \ldots, \mathrm{C}_{2} \mathrm{H}_{2}, \ldots\right)$, but also those which can have a significant influence on the thermal structure of the system (cf. the review on the chemistry of circumstellar envelopes by Tsuji (1986) and references therein). An immediate connection between the occurrence of particular molecules and the onset of dust formation has been pointed out by Stencel (1987), who suggests a scenario of "catastrophic" $\mathrm{SiO}$-formation initiated by $\mathrm{CO}$.

For most applications only chemical equilibrium calculations are available. This assumption is reliable only for systems where the chemistry is essentially determined by kinetic reactions. If a system, however, is contaminated by UV-radiation (e.g. of a chromosphere), the molecular abundances may depart drastically from their 
equilibrium values (cf. Clegg et al., 1983), an effect which is additionally increased by velocity fields (Goeres et al., 1988).

These Non-LTE effects concern the molecular abundances of nearly all components due to ionization and dilution. Hence dust formation and growth could be strongly modified or even prohibited in such environments. However, until now, no methods exist allowing for a consistent quantitative treatment of heterogeneous nucleation in a partially ionized multicomponent system, in particular if grain charging has to be taken into account.

These effects should be important for dust formation in shells of late type stars with chromospheres, in novae ejecta, and in stellar outflows where shock waves play a significant role.

Besides these methodological aspects, in any case the chemistry provides basic constraints for the dust formation process: As a general rule we assume that initial dust formation has to proceed via those molecules of high abundance, which are not blocked to further reactions by high bond energies (like $\mathrm{CO}, \mathrm{N}_{2}, \ldots$ ), and which allow for high temperature condensates. By these arguments most molecules can be ruled out for playing a role in the nucleation process (cf. Gail and Sedlmayr, 1986, $1987 a$ ). However, the result may strongly depend upon the adoption of an equilibrium or a non-equilibrium chemistry, thus suggesting different primary condensates by different approaches.

\section{RESULTS AND DISCUSSION}

Table 1 summarizes various approaches hitherto applied to dust condensation in stellar outflows.

The detailed calculations of dust forming stellar outflows show, that the various approaches of Table 1 can well explain the observed global thermodynamic and hydrodynamic properties of these objects (e.g. temperature structure, velocity field, optical depth, IR excess, ...). However, this is essentially due to the fact that only macroscopic quantities, like the dust absorption coefficient (which depends only on the total amount of condensed material), enter into the equations for radiative transfer and hydrodynamics. For this reason, the resulting global structure of the shell, which is essentially determined by the fact that sufficient dust is present, and not by the particular processes of its formation, allows no definite conclusions concerning the reliability of the adopted condensation theory (cf. Gail and Sedlmayr, $1987 b)$.

Another general feature of classical nucleation theory is that effective cluster nucleation requires the supersaturation ratio $S$ for the condensing species to be much larger than unity ( $S>10$ for C-stars, $S>10^{4}$ for M-stars), thus indicating an extreme non-equilibrium process with a condensation temperature much smaller than the melting (evaporation) temperature of the corresponding solid particle. This condition is met by all homogeneous condensation theories of Table 1 . However, despite a considerable reduction, at least in case of C-stars, the resulting condensation temperature is still too high to be compatible with the observations. This fact casts strong doubts on whether any approach based on classical nucleation theory is appropriate for treating carbon dust formation in C-stars (cf. Salpeter, 
TABle 1: SUMMARY OF CONDENSATION THEORIES

\begin{tabular}{|c|c|c|c|c|}
\hline Objects & $\begin{array}{c}\text { Condensation } \\
\text { Theory }\end{array}$ & $\begin{array}{l}\text { Nucleating } \\
\text { Molecules }\end{array}$ & $\begin{array}{c}\text { Primary } \\
\text { Condensates }\end{array}$ & References $^{\dagger}$ \\
\hline \multirow[t]{3}{*}{$\begin{array}{l}\text { C-stars } \\
\left(\varepsilon_{C}>\varepsilon_{O}\right)\end{array}$} & $\begin{array}{l}\text { homomolecular } \\
\text { homogenous } \\
\text { condensation }\end{array}$ & $C_{1}, C_{2}, C_{3}, \ldots$ & $C_{N}$ & $\begin{array}{l}\text { e.g. Fix (1969), } \\
\text { Tabak et al. (1975), } \\
\text { Salpeter (1974), } \\
\text { Gail and Sedlmayr } \\
(1984,1985,1987 a)\end{array}$ \\
\hline & $\begin{array}{l}\text { heteromolecular } \\
\text { homogeneous } \\
\text { condensation }\end{array}$ & $\begin{array}{l}C_{1}, C_{2}, C_{3}, \ldots \\
C_{2} H_{2}, C_{2} H, \ldots\end{array}$ & $C_{N}$ & $\begin{array}{l}\text { Gail and Sedlmayr } \\
\text { (1988) }\end{array}$ \\
\hline & $\begin{array}{l}\text { chemical } \\
\text { pathway }\end{array}$ & $\mathrm{C}_{2} \mathrm{H}_{2}$ & $\begin{array}{l}\text { PAHs and } \\
\text { large planar } \\
\text { structures }\end{array}$ & $\begin{array}{l}\text { Keller (1987), } \\
\text { Gail and Sedlmayr } \\
(1987 a)\end{array}$ \\
\hline $\begin{array}{c}\text { R-Cor- } \\
\text { Bor-stars } \\
\left(\varepsilon_{C}>\varepsilon_{H}\right)\end{array}$ & $\begin{array}{l}\text { homomolecular } \\
\text { homogeneous } \\
\text { condensation }\end{array}$ & $C_{1}, C_{2}, C_{3}, \ldots$ & $C_{N}$ & $\begin{array}{l}\text { Fadeyev } \\
(1983,1986,1988 b)\end{array}$ \\
\hline \multirow[t]{4}{*}{$\begin{array}{l}\text { M-stars } \\
\left(\varepsilon_{C}<\varepsilon_{O}\right)\end{array}$} & $\begin{array}{l}\text { homomolecular } \\
\text { homogeneous } \\
\text { condensation }\end{array}$ & $\mathrm{SiO}, \mathrm{MgS}, \mathrm{Fe}$ & $\begin{array}{l}(\mathrm{SiO})_{N} \\
(\mathrm{MgS})_{N} \\
(\mathrm{Fe})_{N}\end{array}$ & $\begin{array}{l}\text { Gail and } \\
\text { Sedlmayr (1986) }\end{array}$ \\
\hline & \multirow[t]{3}{*}{$\begin{array}{l}\text { heteromolecular } \\
\text { homogeneous } \\
\text { condensation }\end{array}$} & $\begin{array}{l}\mathrm{SiO}, \mathrm{TiO}, \mathrm{Al} \\
\mathrm{Ca}, \mathrm{Mg}, \mathrm{Fe}\end{array}$ & $\begin{array}{l}\left(\mathrm{Al}_{2} \mathrm{O}_{3}\right)_{N}^{*} \\
\left(\mathrm{CaTiO}_{3}\right)_{N}^{*} \\
\left(\mathrm{MgSiO}_{3}\right)_{N}^{*} \\
(\mathrm{Fe})_{N}\end{array}$ & $\begin{array}{l}\text { Kozasa and } \\
\text { Hasegawa (1977), } \\
\text { Draine (1979) }\end{array}$ \\
\hline & & $\mathrm{SiO}, \mathrm{Mg}, \mathrm{H}_{2} \mathrm{O}$ & $\begin{array}{l}\left(\mathrm{Mg}_{2} \mathrm{SiO}_{4}\right)_{N}^{*} \\
\left(\mathrm{MgSiO}_{3}\right)_{N}^{*}\end{array}$ & Fadeyev $(1987,1988 a)$ \\
\hline & & $\begin{array}{l}\mathrm{Al}_{2} \mathrm{O}_{3}, \mathrm{MgSiO}_{3}^{*} \\
\mathrm{Fe}\end{array}$ & $\begin{array}{l}\left(\mathrm{Al}_{2} \mathrm{O}_{3}\right)_{N} \\
\left(\mathrm{MgSiO}_{3}\right)_{N}^{*} \\
(\mathrm{Fe})_{N}\end{array}$ & $\begin{array}{l}\text { Kozasa, Hasegawa } \\
\text { and Seki (1984) }\end{array}$ \\
\hline
\end{tabular}

* the nominal molecule does not exist in the gas phase (cf. Gail and Sedhmayr (1986)).

$\dagger$ The reference list is incomplete, and further references are in these papers. 
1977; Donn, 1978; Donn and Nuth, 1985) and favours a chemical pathway description which yields a condensation temperature in agreement with the observations (Keller, 1987; Gail and Sedlmayr, 1987a). On the other hand, for M-stars the condensation temperatures derived for $\mathrm{SiO}, \mathrm{MgS}$, an $\mathrm{Fe}$ are consistent with the observations and therefore suggest that classical homogeneous nucleation might be a suitable description, at least for the formation of the primary condensates (Gail and Sedlmayr, 1986).

Nevertheless, a very important question remains in this context. That is whether the nominal molecule of the solid already has to exist in the gas phase (at least as constituent of the nucleating molecules) in order to allow for a homogeneous nucleation process, as is suggested by several authors (cf. Table 1). A clarification of this fundamental problem would provide a much sounder basis for both the understanding of the initial mechanisms of grain formation and for the judgement of the various descriptions proposed.

Hence, despite the huge efforts in the last decades and the impressive progress achieved, the challenge of adequately describing astrophysical grain formation still exists.

\section{REFERENCES}

Allamandola, L. J., Tielens, A. G. G. M., Barker, J. R. 1987, in Nato ASI Series C, Physical Processes in Interstellar Clouds, eds. G. E. Morfill, M. Scholer, (Dordrecht: Reidel), p. 365.

Becker, R., Döring, W. 1935, Ann. Phys., 24, 719.

Castleman, A. W. 1979, Ap. Space Sci., 65, 337.

Clegg, R. E. S., van Ijzendoorn, L. J., Allamandola, L. J. 1983, M. N. R. A. S., $203,125$.

Dominik, C., Gail, H.-P., Sedlmayr, E. 1988, in prep.

Donn, B. 1978, in Protostars and Planets, ed. T. Gehrels, (Arizona: U of A Press), p. 100.

Donn, B., and Nuth, J. A. 1985, Ap. J., 288, 187.

Donn, B., Hecht, J., Khanna, R., Nuth, J. A., Stranz, D., Anderson, A. D. 1981, Surface Sci., 106, 576.

Dorschner, J., Friedemann, C., Gürtler, J., Henning, Th., Wagner, H. 1986, M. N. R. A. S., 218, 37.

Draine, B. T., Salpeter, E. E. 1977, J. Chem. Phys., 67, 2230.

Draine, B. T. 1979, Ap. Space Sci., 65, 313.

Fadeyev, Y. A. 1983, Ap. Space Sci., 95, 357. 1986, in IAU Coll. No. 87, Hydrogen Deficient Stars and Related Objects, eds. K. Hunger, D. Schönberner, N. Kameswara Rao, (Dordrecht: Reidel), p. 441.

1987, in IAU Symp. No. 122, Circumstellar Matter, eds. I. Appenzeller, C. Jordan, (Dordrecht: Reidel), p. 515.

- 1988a, in IAU'Coll. No. 108, Atmospheric Diagnostics of Stellar Evolution, ed. K. Nomoto, (Berlin: Springer), p. 174. $1988 b, M . N$. R. A. S., $238,65$.

Feder, D., Russel, K. C., Lothe, B., Pound, G. M. 1966, Advan. in Phys., $15,111$.

Fix, J. D. 1969, M. N.R. A. S., 146, 37.

Frenklach, M., Clary, D. W., Gardiner, W. C., Stein, S. E. 1984, in Twentieth Symp. (Int.) on Combustion, p. 887.

Gail, H.-P., Sedlmayr, E. 1984, Astr. Ap., 182, 163.

1985, Astr. Ap., 148, 37.

1986, Astr. Ap., 166, 225.

1987 a, in Nato ASI Series C, Physical Process in Interstellar Clouds, eds. G. E. Morfill, M. Scholer, (Dordrecht: Reidel), p. 275.

1987 b, Astr. Ap., $171,197$.

1988, Astr. Ap., in press.

Gail, H.-P., Keller, R., Sedlmayr, E. 1984, Astr. Ap., 133, 320. 
Goeres, A., Henkel, R., Sedlmayr, E., Gail, H.-P. 1988, in Reviews in Modern Astronomy, ed. G. Klare, (Heidelberg: Springer), in press.

Jura, M. 1983, Ap. J., 267, 647 .

Keller, R. 1987, in Nato ASI Series C, Polycyclic Aromatic Hydrocarbons and Astrophysics, eds. A. Léger, L. d'Hendecourt, N. Boccara, (Dordrecht: Reidel), p. 387.

Kozasa, T., and Hasegawa, H. 1987, Prog. Theor. Phys., 77, 1402.

Kozasa, T., Hasegawa H., Seki, J. 1984, Ap. Space Sci., 98, 61.

Koike, C., Hasegawa, H., Manabe, A. 1980, Ap. Space Sci., 67, 495.

Léger, A., Puget, J. L. 1984, Astr. Ap., 137, L5.

Nuth, J. A., Donn, B. 1987, in IAU Symp. No. 122, Circumstellar Matter, eds. I. Appenzeller, C. Jordan, (Dordrecht: Reidel), p. 559 .

Rengarajan, T. N., Fazio, G. G., Maxson, C. W., McBreen, B., Serio, S., Sciortino, S. 1985, Ap. $J ., 289,630$.

Rossi, S. C. F., and Benevides-Soares, P. 1988, Astr. Ap., $192,379$.

Rowan-Robinson, M., Harris, S. 1983, M. N. R. A.S., 202, 797.

Salpeter, E. E. 1974, Ap. J., 193, 579.

. 1977, Ann. Rev. Astr. Ap., 15, 267.

Stencel, R. E. 1987, in IAU Symp. No. 12R, Circumstellar Matter, eds. I. Appenzeller, C. Jordan, (Dordrecht: Reidel), p. 529 .

Tabak, R. G., Hirth, J. P., Meyrick, G., Roark, T. P. 1975, Ap. J., 196, 457.

Tanabe, T., Ňakada, Y., Kamijo, F. 1983, Pub. Astr. Soc. Japan, 35, 397.

Tielens, A. G. G. M., Allamandola, L. J. 1987, in Nato ASI Series C, Physical Processes in Interstellar Clouds, eds. G. E. Morfill, M. Scholer, (Dordrecht: Reidel), p. 333.

Tielens, A. G. G. M. 1988, in Carbon in the Galaxy: Studies from Earth and Space, ed. J. Tarter, NASA CP, in press.

Tsuji, T. 1986, Ann. Rev. Astr. Ap., 24, 89.

Wirsich, J. 1987, Infrared Phys., 27, 399.

Yamamoto, T., Nishida, S. 1977, Prog. Theor. Phys., 57, 1939. 


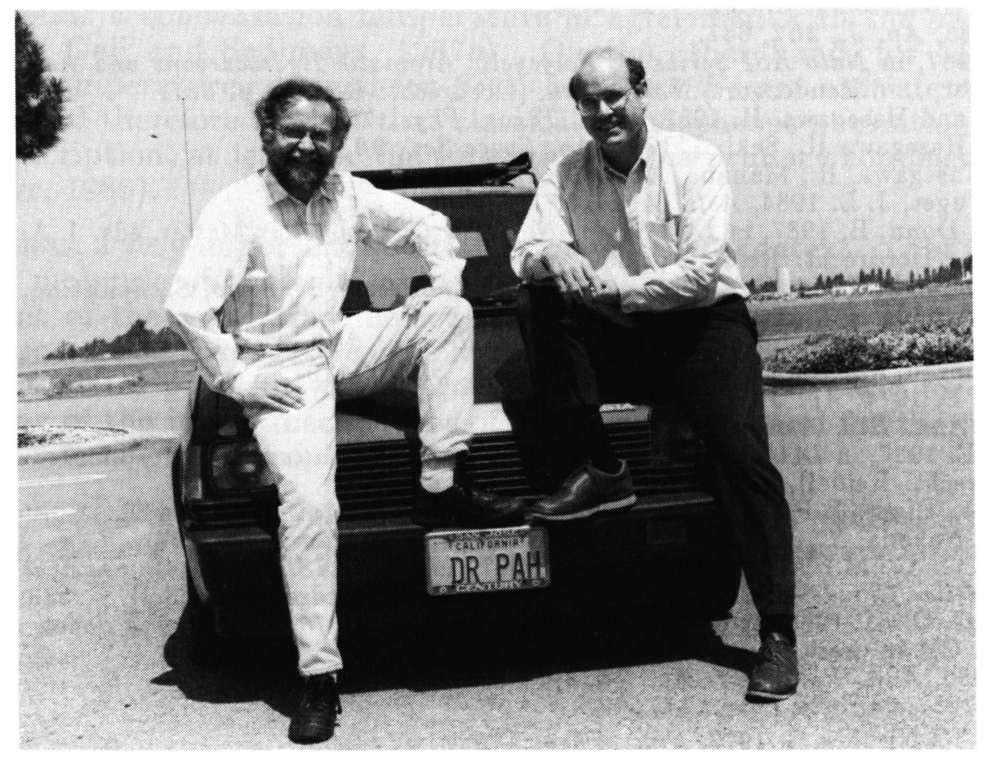

Xander Tielens and Lou Allamandola. 